\title{
Inalador dosimetrado com espaçador artesanal versus nebulizador no tratamento da crise de sibilância na criança
}

\author{
Metered-dose inhalers with home-made spacers versus nebulizers \\ to treat moderate wheezing attacks in children
}

\author{
Liana Consuelo Santana Vilarinho' ${ }^{1}$, Carlos Maurício Cardeal Mendes ${ }^{2}$, Leda Solano de Freitas Souza ${ }^{3}$
}

\section{Resumo}

Objetivo: Realizou-se um ensaio clínico objetivando comparar duas modalidades de administração de salbutamol na crise de sibilância em crianças - através de nebulizador de jato e através de inalador dosimetrado acoplado a um espaçador artesanal.

Material e métodos: Foi estudada uma amostra de conveniência de crianças em crise moderada de sibilância, alocadas aleatoriamente em dois grupos de tratamento, de acordo com o dispositivo inalatório utilizado para a administração de salbutamol (nebulizador - grupo NEB, ou inalador dosimetrado com espaçador - grupo IDE). Os parâmetros utilizados para comparação entre os grupos foram agrupados em tabela de pontos e consistiram em sinais clínicos habitualmente usados na avaliação da gravidade da crise asmática (nível de consciência, coloração da pele, intensidade da dispnéia, intensidade da tiragem, tempo expiratório, entrada de ar e sibilância) e a saturação transcutânea de oxigênio, obtidos antes do tratamento e 15 minutos após cada administração do salbutamol. Como dados adicionais, mediram-se os tempos de preparo e de uso das medicações, computaram-se os custos envolvidos nas duas formas de tratamento, e interrogaram-se os acompanhantes sobre o grau de satisfação em relação aos tratamentos. Cada criança usou salbutamol em doses repetidas a cada 20 minutos, interrompendo-se a administração quando a crise passava de moderada a leve. Quando a crise persistia moderada, após a segunda dose de salbutamol, acrescentava-se corticóide oral. A participação das crianças era concluída quando elas passavam à crise leve, ou após a terceira dose de salbutamol. A dose de salbutamol usada no grupo IDE foi $1 / 3$ da dose usada no grupo NEB.

Resultados: Participaram do estudo 54 crianças com idades que variaram de 22 dias a 11,7 anos. Cada grupo abrangeu 27 crianças. Os grupos não diferiram demograficamente e em relação à pontuação clínica e aos valores de oximetria. A comparação dos parâmetros clínicos e de saturação de oxigênio, entre os grupos, não mostrou diferença significativa após as doses de salbutamol, havendo equivalência clínica entre doses diferentes (três vezes menor no grupo IDE, em relação ao grupo NEB); os tempos de preparo e administração da medicação e o custo do tratamento foram significativamente menores no grupo IDE. O grau de satisfação das famílias foi semelhante nos dois grupos.

Conclusão: $\mathrm{O}$ inalador dosimetrado com espaçador artesanal pode ser utilizado na administração de salbutamol a crianças em crise de sibilância, com algumas vantagens em relação ao nebulizador.

J Pediatr (Rio J) 2003;79(5):403-12: Espaçador artesanal, inalador dosimetrado, crise de sibilância, criança.

\section{Abstract}

Objective: To compare the effectiveness of salbutamol administration by metered-dose inhaler with a home-made spacer versus jet nebulizer in children with moderate wheezing attacks.

Material and methods: A randomized, single-blinded trial was performed with a convenience sample of children presenting wheezing. The children were enrolled in an emergency room and randomly assigned to one of two treatment groups: home-made spacer group or nebulizer group. Clinical scores and oxygen saturation were recorded at baseline and 15 minutes after salbutamol administration. Treatment with salbutamol $(100 \mu \mathrm{g} / 3 \mathrm{~kg}$ in the spacer group, and $250 \mu \mathrm{g} / 3 \mathrm{~kg}$ in the nebulizer group) was repeated at 20-minute intervals, until the child was considered to have improved significantly, with no need of any further dose, or until three doses were delivered. Treatment cost, time spent to prepare and deliver the drug, and level of parental satisfaction with the treatment were recorded.

Results: Fifty-four children with age between 22 days and 11.7 years were enrolled - 27 in each group. Baseline and demographic characteristics were similar for both groups. The spacer was as effective as the nebulizer in terms of clinical score and oxygen saturation. The different doses $(100 \mu \mathrm{g} / 3 \mathrm{~kg}$ with the spacer, and $250 \mu \mathrm{g} / 3 \mathrm{~kg}$ with the nebulizer) were shown to be clinically equivalent. Treatment cost was significantly lower in the spacer group, as was the time to prepare and deliver the drug. Parental satisfaction was similar for both inhaler devices.

Conclusion: The home-made spacer with a metered-dose inhaler is a cost-effective alternative to a jet nebulizer in the delivery of salbutamol to children with moderate wheezing attacks.

J Pediatr (Rio J) 2003;79(5):403-12: Home-made spacer, metereddose inhaler, acute asthma, children.

1. Mestrado em Pediatria (UFBA). Professora colaboradora do Dep. de Pediatria da Faculdade de Medicina da UFBA.

2. Doutorado em Saúde Coletiva - concentração em Epidemiologia. Médico estatista do Instituto de Saúde Coletiva (UFBA) e Professor de Saúde Coletiva e Estatística da Escola Baiana de Medicina.

3. Doutorado em Medicina (UFBA). Professora Adjunta do Dep. de Pediatria da Faculdade de Medicina da UFBA. Chefe do Setor de Pneumologia Pediátrica do Centro Pediátrico Prof. Hosannah de Oliveira (UFBA).

Artigo submetido em 15.04.02, aceito em 11.06.03. 


\section{Introdução}

Os broncodilatadores são rotineiramente ministrados por via inalatória no tratamento da crise asmática. Em crianças, os nebulizadores de jato são os dispositivos mais freqüentemente usados para esse fim. No entanto, eles são desconfortáveis, particularmente para os lactentes ${ }^{1,2}$, demandam tempo de 15 a 20 minutos para a administração da dose prescrita, e necessitam de uma fonte de oxigênio ou ar comprimido para gerar o aerossol. O fluxo de oxigênio, a distância face/máscara, o volume corrente, a frequiência respiratória e a técnica inalatória do paciente determinam variação na deposição, em vias aéreas inferiores, de 3 a $13 \%$ do total das partículas disponíveis para inalação ${ }^{3-5}$.

Os inaladores dosimetrados podem ser utilizados eficientemente em todas as idades, durante a respiração normal, desde que acoplados a um espaçador ${ }^{5-7}$, e permitem ministração rápida da medicação, sendo assim melhor tolerados por crianças pequenas; além disso, em torno de $20 \%$ das partículas geradas atingem as vias aéreas inferiores ${ }^{5,8-12}$, com padrão de deposição mais previsível e menos variável que os nebulizadores. Apesar dessas vantagens, os inaladores dosimetrados com espaçadores ainda não substituíram os nebulizadores na maioria dos serviços de emergência e hospitais pediátricos. Diversos tipos de espaçadores industrializados estão disponíveis para uso pediátrico; têm vários tamanhos e formas, acoplam-se ao paciente através de máscara ou de terminação bucal, e alguns possuem válvulas inalatórias. O custo dificulta seu uso generalizado. Por essa razão, observa-se, na prática médica, a improvisação de espaçadores (a partir de garrafas ou outros utensílios plásticos); poucos trabalhos estudaram a eficácia dos espaçadores alternativos, de fabricação artesanal ${ }^{13-16}$. Um dos estudos ${ }^{16}$ compara um espaçador convencional (industrializado), um de garrafa plástica com boa adesão, outro sem adesão completa à face do paciente, e um espaçador feito de copo plástico; os dois primeiros tiveram efeitos semelhantes e superiores aos demais, tendo o espaçador de copo mostrado o pior desempenho.

O objetivo principal deste trabalho é comparar a eficiência clínica do inalador dosimetrado com espaçador artesanal à do nebulizador de jato, na terapia broncodilatadora, em crianças em crise de sibilância; secundariamente, pretende-se comparar as duas modalidades terapêuticas, relativamente a custos, tempo de preparo e uso da medicação e grau de satisfação das famílias.

\section{Material e métodos}

\section{Delineamento do estudo}

Ensaio clínico, com alocação aleatória dos pacientes em dois grupos de tratamento, e avaliação "cega" dos resultados terapêuticos. A população-alvo foi a de crianças em crise de sibilância atendidas no setor de pronto-atendimento (PA) do Centro Pediátrico Prof. Hosannah de Oliveira (CPPHO- UFBA). O estudo foi realizado em duas salas: $\mathrm{em}$ uma delas ficava o avaliador - o mesmo para todas as crianças (sala 1), e na outra o ministrador do broncodilatador (sala 2). O avaliador recebia as crianças, realizava a primeira avaliação e incluía as crianças no estudo; o ministrador alocava as crianças nos grupos e ministrava a primeira dose; sucediam-se, então, avaliações e ministrações de doses nas salas respectivas (Figura 1).

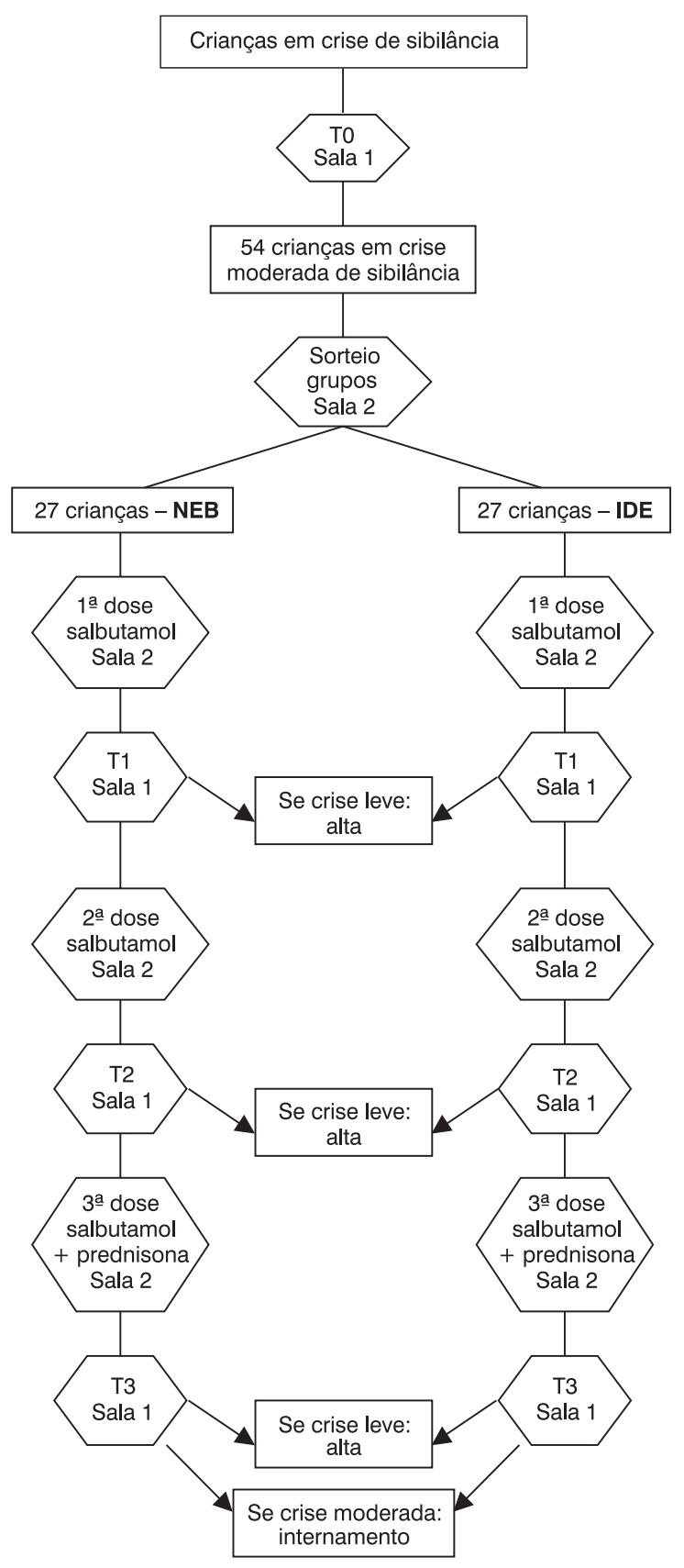

Figura 1 - Fluxograma do estudo (T0, T1, T2 e T3 são momentos de avaliação, alta e internamento são momentos de finalização do estudo) 


\section{Amostra estudada}

Durante quatro meses, em duas tardes por semana, foram selecionados pacientes (amostra de conveniência). Os critérios de inclusão foram: meninos e meninas de até 12 anos, em demanda espontânea ao PA por crise de sibilância moderada, com consentimento verbal dos pais, para participação no estudo, após informação sobre a natureza do mesmo. Excluíram-se os que estavam usando broncodilatadores ou corticosteróides e os que tinham febre, ou doenças crônicas (refluxo gastroesofágico, fibrose cística, cardiopatias congênitas, deficiências imunológicas, etc.) segundo seus familiares.

\section{Formação dos grupos}

Após a inclusão de cada criança, seu acompanhante sorteava um envelope com o nome do grupo (IDE ou NEB). Havia quatro envelopes com a sigla IDE e quatro com a sigla NEB. Os envelopes sorteados eram mantidos à parte até que os oitos fossem sorteados, quando então eram outra vez reunidos para os próximos sorteios, garantindo-se, assim, um número igual de crianças nos dois grupos.

\section{Regimes terapêuticos}

- Grupo IDE: utilizou salbutamol em inalador dosimetrado, com liberação de $100 \mu \mathrm{g}$ por disparo $\left(\right.$ Aerolin $\left.^{\circledR}\right)$. O inalador dosimetrado era acoplado a espaçadores confeccionados no CPPHO, a partir de frascos de soro. Perfuravase o fundo do frasco no formato do bocal do inalador, para permitir o encaixe justo deste último; seccionava-se a outra extremidade do frasco transversalmente, revestindo a borda do corte com esparadrapo, para amaciar o contato com a pele do rosto das crianças, obtendo o melhor ajuste possível. Não havia válvula respiratória e não foi utilizada máscara facial nem pasta de silicone para selar o contato entre rosto e espaçador; o espaçador era colocado ao redor da boca da criança sem englobar o nariz, que era pinçado pelos dedos do ministrador (Figura 2). Dessa maneira, evitou-se a perda de partículas que se depositariam nas tortuosidades das cavidades nasais; foram produzidos espaçadores com $250 \mathrm{ml} \mathrm{e} \mathrm{com} 500 \mathrm{ml}$ para crianças menores de 3 anos e de 3 a 12 anos, respectivamente. Esses volumes correspondem a cerca de 2 ou 3 vezes o volume corrente das crianças (cerca de $7 \mathrm{ml} / \mathrm{kg}$ ) ${ }^{5}$. A cada criança ministraramse $100 \mu \mathrm{g}$ (um disparo) de salbutamol para cada $3 \mathrm{~kg}$ de peso, um disparo por vez, a cada 20 segundos, esperandose a criança realizar três inalações antes de afastar o espaçador; às crianças colaborativas, solicitava-se que realizassem inspiração profunda logo após cada disparo do inalador e prendessem a respiração por 10 segundos. Agitava-se o inalador vigorosamente entre os disparos.

- Grupo NEB: usou salbutamol em solução contendo $250 \mu \mathrm{g} /$ gota $\left(\right.$ Aerolin $\left.^{\circledR}\right)$, na dose de $250 \mu \mathrm{g}$ (1 gota) para cada $3 \mathrm{~kg}$ de peso, em $5 \mathrm{ml}$ de soro fisiológico, no nebulizador de jato, com fluxo de oxigênio de $81 / \mathrm{min}$. As crianças inalavam através de respiração normal, durante 15 minutos.

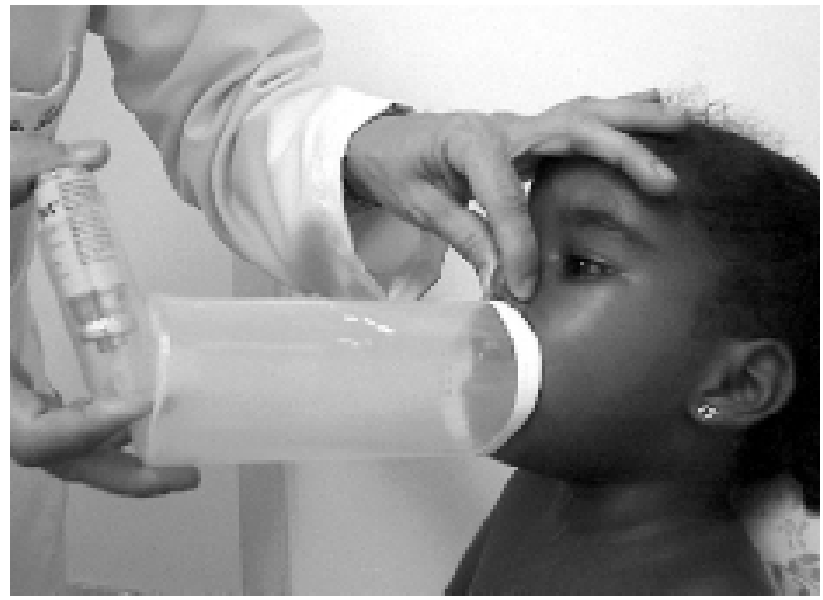

Figura 2 - Espaçador artesanal usado no setor de pronto-atendimento do Centro Pediátrico Prof. Hosannah de Oliveira e neste estudo

\section{Avaliação da gravidade da crise}

Os parâmetros de comparação entre os grupos foram sinais clínicos habitualmente usados na avaliação da gravidade da crise asmática (nível de consciência, coloração da pele, intensidade da dispnéia e da tiragem, tempo expiratório, entrada de ar e sibilância) e a saturação transcutânea de oxigênio. Esses parâmetros compõem uma tabela de pontos (Tabela 1). As avaliações foram realizadas nos momentos de chegada ao PA (tempo zero - T0) e 15 minutos após cada dose de salbutamol (T1, T2, T3). Considerou-se em crise moderada as crianças com pontuação entre 12 e 16 . As pontuações nos diversos tempos foram comparadas entre os grupos, como indicadores da eficácia dos dispositivos inalatórios.

\section{Outros parâmetros de comparação}

O tempo de preparo das medicações e o tempo de uso foram registrados na sala 2 .

Após o término do estudo, foram calculados os custos do tratamento de cada paciente, utilizando-se as informações de preços fornecidas pelo setor de compras do CPPHO, conforme descrito a seguir.

No grupo NEB:

- custo de salbutamol: preço do frasco dividido pelo número de gotas contidas versus número de gotas consumido;

- custo de soro fisiológico: preço do frasco de soro fisiológico dividido pelo volume do frasco versus volume consumido;

- custo do oxigênio: preço do metro cúbico do oxigênio versus quantidade consumida. 
No grupo IDE:

- custo de salbutamol: preço do medicamento dividido por 200 (número de disparos ou doses de um tubo do medicamento) versus número de disparos efetuados;

- os espaçadores não foram contabilizados, uma vez que foram confeccionados a partir de material que seria descartado;

- não foram contabilizados, também, o trabalho dos profissionais de enfermagem e o tempo de permanência no PA.

Tabela 1 - Tabela de pontuação para avaliação da gravidade da crise de sibilância (CPPHO)

\begin{tabular}{|c|c|c|}
\hline Parâmetros & Categorias & Pontos \\
\hline Consciência & $\begin{array}{l}\text { Normal } \\
\text { Agitada ou deprimida } \\
\text { Torporosa }\end{array}$ & $\begin{array}{l}1 \\
2 \\
3\end{array}$ \\
\hline Pele (coloração) & $\begin{array}{l}\text { Normal } \\
\text { Pálida } \\
\text { Cianótica (ar) }\end{array}$ & $\begin{array}{l}1 \\
2 \\
3\end{array}$ \\
\hline Tiragem costal & $\begin{array}{l}\text { Leve } \\
\text { Moderada } \\
\text { Acentuada }\end{array}$ & $\begin{array}{l}1 \\
2 \\
3\end{array}$ \\
\hline Dispnéia & $\begin{array}{l}\text { Leve } \\
\text { Moderada } \\
\text { Acentuada }\end{array}$ & $\begin{array}{l}1 \\
2 \\
3\end{array}$ \\
\hline $\begin{array}{l}\text { Prolongamento } \\
\text { expiratório }\end{array}$ & $\begin{array}{l}\text { Leve } \\
\text { Moderado } \\
\text { Acentuado }\end{array}$ & $\begin{array}{l}2 \\
3\end{array}$ \\
\hline Entrada de ar & $\begin{array}{l}\text { Bem distribuída } \\
\text { Diminuída em algumas áreas } \\
\text { Difusamente diminuída }\end{array}$ & $\begin{array}{l}1 \\
2 \\
3\end{array}$ \\
\hline Sibilância & $\begin{array}{l}\text { Só na expiração } \\
\text { Na expiração e na inspiração } \\
\text { Abolida }\end{array}$ & $\begin{array}{l}1 \\
2 \\
3\end{array}$ \\
\hline Oximetria & $\begin{array}{l}>95 \% \\
\text { de } 91 \text { a } 95 \% \\
<91 \%\end{array}$ & $\begin{array}{l}1 \\
2 \\
3\end{array}$ \\
\hline Classificação & $\begin{array}{l}\text { Crise leve } \\
\text { Crise moderada } \\
\text { Crise grave } \\
\text { Crise muito grave }\end{array}$ & $\begin{array}{l}3 \text { a } 11 \text { pontos } \\
2 \text { a } 16 \text { pontos } \\
7 \text { a } 22 \text { pontos } \\
3 \text { a } 24 \text { pontos }\end{array}$ \\
\hline
\end{tabular}

Na investigação sobre o grau de satisfação dos acompanhantes usou-se um método de fácil compreensão, levando em consideração o nível educacional primário dos acompanhantes das crianças estudadas - todas de classe socioeconômica baixa. No final do tratamento, perguntava-se a cada acompanhante o que tinha achado do tratamento oferecido; a resposta era através da escolha de uma dentre três figurinhas com expressões faciais sorridente, zangada ou neutra.

\section{Finalização do estudo}

Cada criança usou doses de salbutamol repetidas a cada 20 minutos, interrompendo-se a administração quando a crise passava de moderada a leve. Quando persistia moderada após a segunda dose de salbutamol, acrescentava-se corticóide oral. A participação das crianças terminava se passassem a crise leve, ou após a terceira dose de salbutamol, ou se apresentassem agravamento. As crianças que necessitavam continuar o tratamento após a terceira dose eram mantidas no PA, aos cuidados da equipe de plantão.

\section{Análise estatística}

A carência de dados de comparação entre nebulizador e inalador dosimetrado com espaçador artesanal, na literatura, impossibilitou o cálculo do tamanho amostral para este estudo. Os dois grupos foram comparados entre si, através de testes não paramétricos, porque a maioria das variáveis estudadas não aderiu à distribuição normal: teste de MannWhitney, para as pontuações nos diversos tempos de avaliação, tempos de preparo e ministração das medicações e custos do tratamento; nas comparações das variáveis categóricas, usou-se o teste do qui-quadrado ou o de Fisher. O poder das comparações foi calculado após o término do estudo, durante a análise dos dados (Tabela 2), para teste paramétrico (teste $t$ de Student), usando-se as médias, desvios padrão e o número de crianças. Em seguida, foi corrigido para teste não paramétrico (teste de Mann-Whitney), tomando-se o valor de $95 \%$ do poder calculado, de acordo com Siegel ${ }^{17}$. Utilizou-se o SAS (Statistical Analysis System, Copyright by SAS Institute, Cary, NC, USA, version 6.12).

Tabela 2 - Poder dos testes de comparação entre os grupos NEB e IDE das variáveis pontuação global, oximetria e frequiência respiratória, nos quatro momentos de avaliação (basal - T0, e após cada dose de salbutamol - T1, T2, e T3) de crianças em crise de asma, no CPPHO. Os valores foram calculados após a realização do estudo

\begin{tabular}{cccc}
\hline $\begin{array}{c}\text { Momentos } \\
\text { de avaliação }\end{array}$ & $\begin{array}{c}\text { Pontuação } \\
\text { global }\end{array}$ & $\begin{array}{c}\text { Poder (\%) } \\
\text { Oximetria }\end{array}$ & $\begin{array}{c}\text { Frequiência } \\
\text { respiratória }\end{array}$ \\
\hline T0 & 5,50 & 6,70 & 10,56 \\
T1 & 19,86 & 7,00 & 4,80 \\
T2 & 15,03 & 10,50 & 7,53 \\
T3 & 15,12 & 20,89 & 6,02 \\
Média & 13,88 & 11,27 & 7,23 \\
\hline
\end{tabular}

\section{Aspectos éticos}

O estudo foi submetido à Comissão de Ética em Pesquisa do Hospital Universitário Prof. Edgard Santos e aprovado. Foi obtido consentimento informado verbal dos responsáveis pelas crianças. 


\section{Resultados}

Estudaram-se 27 pacientes em cada grupo, 30 meninos (14 no grupo IDE e 16 no grupo NEB) e 24 meninas (13 no IDE e 11 no NEB). Duas crianças no IDE (idades de 6 meses e 2 anos, respectivamente) e 5 crianças no NEB (idades de 22 dias, 3 meses, 12 meses -2 crianças - e 4 anos) nunca haviam tido outras crises de sibilância. Características demográficas, freqüências respiratórias e cardíacas, pontuação global e valores de oximetria, na chegada ao PA, estão na Tabela 3. Os grupos não diferiram significativamente, exceto em relação à freqüência cardíaca na avaliação inicial, superior no NEB $(\mathrm{p}<0,03)$.

A Tabela 4 mostra a estatística descritiva para as variáveis de avaliação, nos tempos T1, T2 e T3, nos grupos. Os grupos não diferiram significativamente (Tabela 5), exceto quanto à entrada de ar, com pontuação menor no IDE.

Houve uma maior dispersão das pontuações no IDE, no tempo 0; ao contrário, nos demais tempos, observou-se maior concentração das pontuações no grupo IDE e maior dispersão no grupo NEB (Figura 3).

Nenhum paciente piorou durante o estudo. Após a segunda dose de salbutamol, 8 (29,6\%) pacientes do NEB e $6(22,2 \%)$ do IDE tiveram alta, e após a terceira dose, 11 $(40,7 \%)$ pacientes do NEB e $16(59,3 \%)$ do IDE tiveram alta (diferenças não significativas). Após a segunda dose de salbutamol, $15(55,6 \%)$ pacientes do NEB e $12(44,4 \%)$ do IDE necessitaram de corticoterapia $(\mathrm{p}=0,076)$. Permaneceram no PA 8 pacientes do NEB e 5 do IDE $(\mathrm{p}=0,19)$.
O custo total dos tratamentos foi de $\mathrm{R} \$ 51,47$ - média de $\mathrm{R} \$ 0,70$ por dose (US $\$ 14,5$ - média de US $\$ 0,20$ ), no NEB, e de $\mathrm{R} \$ 11,32$ - média de $\mathrm{R} \$ 0,15$ por dose (US\$ 3,20média de US\$ 0,04), no IDE ( $\mathrm{p}=0,0001)$ : o custo do tratamento no IDE representou $22 \%$ do custo no NEB.

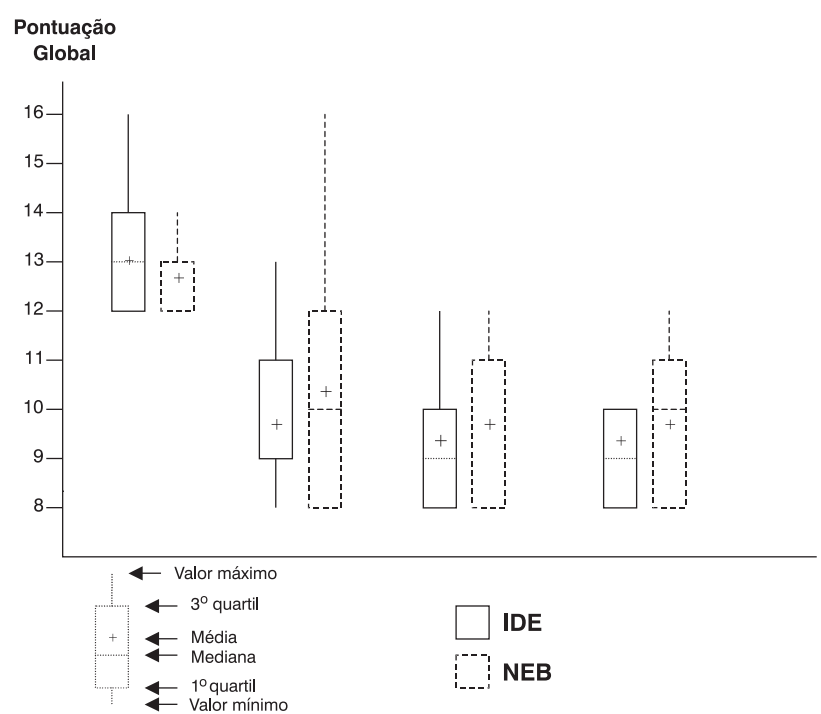

Figura 3 - Distribuição da pontuação global, nos tempos T0, T1, $\mathrm{T} 2$ e T3, nos dois grupos de tratamento (IDE-inalador dosimetrado com espaçador e NEB - nebulizador de jato)

Tabela 3 - Características demográficas, freqüências respiratórias e frequiências cardíacas, pontuação global e valores de oximetria, na chegada ao PA, em 54 crianças em crise de sibilância, atendidas no CPPHO

\begin{tabular}{lccc}
\hline Características dos 54 pacientes & $\begin{array}{c}\text { Grupo NEB* } \\
(\mathbf{n}=\mathbf{2 7})\end{array}$ & $\begin{array}{c}\text { Grupo IDE } \dagger \\
(\mathbf{n}=\mathbf{2 7})\end{array}$ & Valor de p \\
\hline Idade $\geq 2$ anos & 14 & 18 & 0,78 \\
Idade $<2$ anos & 13 & 9 & \\
Sexo masculino & 16 & 14 & 0,58 \\
Sexo feminino & 11 & 13 & \\
Frequiência cardíaca - T0 & 137 & 120 & 0,03 \\
(batimentos/min: média e variação) & $(123-146)$ & $(112-140)$ & \\
Freqüûncia respiratória - T0 & 52 & 48 & 0,39 \\
(incursões/min: média e variação) & $(44-62)$ & $(40-60)$ & \\
Pontuação global - T0 & 13 & 13 & 0,89 \\
(mediana e variação) & $(12-16)$ & $(12 \mathrm{e} 16)$ & \\
Oximetria - T0 & 94 & 94 & 0,74 \\
(\%: mediana e variação) & $(85-99)$ & $(87-98)$ & \\
\hline
\end{tabular}

* Grupo NEB = grupo que usou salbutamol através de nebulizador de jato.

$\dagger$ Grupo IDE = grupo que usou salbutamol através de inalador dosimetrado e espaçador.

T0 = tempo zero (basal) de avaliação da gravidade da crise - antes do tratamento. 
Tabela 4 - Estatística descritiva para as variáveis usadas na avaliação da gravidade da crise de sibilância em 54 crianças, no CPPHO, nos tempos T0, T1, T2 e T3, nos dois grupos de tratamento

\begin{tabular}{|c|c|c|c|c|c|c|c|c|c|}
\hline \multirow[t]{2}{*}{ Variáveis } & \multirow[t]{2}{*}{ Estatística } & \multicolumn{4}{|c|}{ Grupo NEB* } & \multicolumn{4}{|c|}{ Grupo IDE ${ }^{\dagger}$} \\
\hline & & T0 & $\mathbf{T 1}$ & $\mathbf{T 2}$ & T3 & T0 & $\mathbf{T 1}$ & $\mathbf{T} 2$ & $\mathbf{T 3}$ \\
\hline \multirow[t]{7}{*}{ Pontuação global } & Média & 12,88 & 10,44 & 9,59 & 9,73 & 12,96 & 9,85 & 9,14 & 9,28 \\
\hline & $\mathrm{DP} \doteqdot$ & 1,08 & 2,08 & 2,13 & 2,05 & 1,16 & 1,68 & 1,23 & 1,34 \\
\hline & Mínimo & 12 & 8 & 8 & 8 & 12 & 8 & 8 & 8 \\
\hline & Quartil 1 & 12 & 8 & 8 & 8 & 12 & 9 & 8 & 8 \\
\hline & Mediana & 13 & 10 & 8 & 10 & 13 & 9 & 9 & 9 \\
\hline & Quartil 3 & 13 & 12 & 11 & 11 & 14 & 11 & 10 & 10 \\
\hline & Máximo & 16 & 16 & 17 & 16 & 16 & 15 & 12 & 14 \\
\hline \multirow[t]{7}{*}{ Oximetria (\%) } & Média & 93,96 & 95,62 & 95,66 & 95,26 & 93,62 & 95,29 & 96,18 & 96,14 \\
\hline & $\mathrm{DP} \ddagger$ & 3,02 & 2,92 & 3,13 & 3,10 & 2,93 & 2,44 & 2,11 & 2,30 \\
\hline & Mínimo & 85 & 86 & 82 & 85 & 87 & 90 & 90 & 89 \\
\hline & Quartil 1 & 93 & 95 & 95 & 94 & 92 & 93 & 95 & 95 \\
\hline & Mediana & 94 & 96 & 96 & 96 & 94 & 96 & 97 & 96 \\
\hline & Quartil 3 & 96 & 98 & 97 & 97 & 96 & 97 & 97 & 98 \\
\hline & Máximo & 99 & 99 & 99 & 99 & 98 & 99 & 99 & 99 \\
\hline \multirow{7}{*}{$\begin{array}{l}\text { Freqüência } \\
\text { respiratória } \\
\text { (incursões/min) }\end{array}$} & Média & 52,0 & 46,3 & 42,9 & 43,2 & 49,6 & 46,1 & 44,6 & 42,2 \\
\hline & $\mathrm{DP} \doteqdot$ & 11,34 & 12,90 & 12,14 & 9,76 & 13,10 & 13,46 & 12,77 & 11,74 \\
\hline & Mínimo & 28 & 23 & 20 & 24 & 25 & 22 & 25 & 20 \\
\hline & Quartil 1 & 44 & 36 & 30 & 36 & 40 & 36 & 36 & 35 \\
\hline & Mediana & 52 & 44 & 44 & 46 & 48 & 44 & 44 & 40 \\
\hline & Quartil 3 & 62 & 60 & 50 & 52 & 60 & 56 & 52 & 52 \\
\hline & Máximo & 68 & 68 & 71 & 60 & 72 & 68 & 74 & 60 \\
\hline
\end{tabular}

\footnotetext{
* Grupo NEB: grupo que usou salbutamol através de nebulizador de jato, † Grupo IDE: grupo que usou salbutamol através de inalador dosimetrado e espaçador, ‡ DP: desvio padrão.

TO: tempo zero (basal) de avaliação da gravidade da crise.

T2: momento de avaliação após a segunda dose de salbutamol.

T1: momento de avaliação após a primeira dose de salbutamol.

T3: momento de avaliação após a terceira dose de salbutamol.
}

O tempo total gasto no preparo da medicação foi de 6.720 segundos (média de 92 segundos por dose preparada) no NEB, e de 1.948 segundos (média de 26 segundos por dose) no IDE ( $\mathrm{p}=0,0001)$; gastou-se, no IDE, $28,3 \%$ do tempo no NEB. O tempo total gasto na ministração da medicação foi de 66.840 segundos (916 segundos por dose) no NEB, e de 9.239 segundos (123 segundos por dose) no IDE ( $\mathrm{p}<0,0001)$; no IDE, o tempo de ministração da medicação correspondeu a $13,4 \%$ do tempo gasto no NEB.

A distribuição das respostas sobre satisfação com o tratamento foi semelhante nos dois grupos: 21 famílias no NEB e 22 no IDE escolheram a figura sorridente, quatro no NEB e cinco no IDE escolheram a figura neutra e duas no NEB e nenhuma no IDE escolheram a figura zangada.

\section{Discussão}

Este é um estudo preliminar, uma vez que a escassez de dados de comparação entre os dois tipos de dispositivos inalatórios, na literatura, impossibilitou o cálculo de tamanho amostral e a definição do poder dos testes estatísticos, previamente à sua realização. Os principais trabalhos existentes ${ }^{13-16,18-23}$ têm metodologias variadas, utilizando diferentes espaçadores e parâmetros (Tabela 6).
Tabela 5 - Valores de $p$ obtidos para a comparação entre os grupos NEB e IDE, em cada momento de avaliação da crise de sibilância de 54 crianças atendidas no CPPHO

\begin{tabular}{lcccc}
\hline Variáveis & T0 & T1 & T2 & T3 \\
\hline Pontuação global & 0,89 & 0,28 & 0,80 & 0,55 \\
Saturação de O $^{2}$ & 0,74 & 0,50 & 0,44 & 0,29 \\
Frequiência respiratória & 0,39 & 1,00 & 0,74 & 0,93 \\
Frequiência cardíaca & $0,03^{*}$ & 0,21 & 0,24 & 0,06 \\
Consciência & 0,48 & 0,16 & 0,55 & 0,94 \\
Pele (coloração) & $0,04^{*}$ & 0,30 & 0,07 & 0,29 \\
Tiragem costal & 0,23 & 0,41 & 0,56 & 0,22 \\
Dispnéia & 0,34 & 0,74 & 0,70 & 0,37 \\
Entrada de ar & 0,44 & 0,15 & $0,04^{*}$ & 0,94 \\
Prolongamento expiratório & 0,22 & 0,27 & 0,30 & 0,49 \\
Sibilos & 0,10 & 0,48 & 0,22 & 0,61 \\
Peso & 0,27 & - & - & - \\
Idade & 0,78 & - & - & - \\
\hline
\end{tabular}

Grupo NEB = grupo que usou salbutamol através de nebulizador de jato. Grupo IDE = grupo que usou salbutamol através de inalador dosimetrado e espaçador.

T0 = tempo zero (basal) de avaliação da gravidade da crise.

$\mathrm{T} 1$ = momento de avaliação após a primeira dose de salbutamol.

$\mathrm{T} 2$ = momento de avaliação após a segunda dose de salbutamol.

T3 = momento de avaliação após a terceira dose de salbutamol.

* diferenças estatisticamente significantes (as três com pontuação menor no grupo IDE). 
O presente estudo usou uma amostra de conveniência, visando a avaliar os dispositivos inalatórios utilizados no setor de pronto-atendimento do CPPHO; difere dos demais artigos, por avaliar o desempenho de um espaçador feito de frascos de soro. Pode-se prever uma perda de aerossol com esse dispositivo (sem válvula e sem selo no contato com a face). Zar ${ }^{15}$, através de medidas de volume expiratório forçado no primeiro segundo e de pico de fluxo expiratório, mostrou broncodilatação significativamente maior entre crianças que usaram espaçador de garrafa selado em relação às que usaram garrafa não selada. No entanto, no presente estudo, a comparação do aerossol dosimetrado e espaçador foi feita com o nebulizador, usado com máscara facial, não selada, por conseguinte, também com perda de aerossol.

O trabalho incluiu toda a faixa etária atendida no setor de pronto-atendimento, o que o aproximou da "vida real" e o valorizou. Nos menores de três anos, várias condições clínicas podem causar crises asmatiformes; por isso, decidiu-se utilizar o termo crise de sibilância, sem a pretensão de definir rigorosamente o diagnóstico de asma. Vale ressaltar o caráter recidivante das crises em quase todas as crianças. A inclusão de lactentes pequenos aumentou a importância deste estudo, que mostrou a eficácia do inalador dosimetrado com espaçador caseiro, também nessa faixa etária.

A qualidade da avaliação foi garantida pelo cuidado de alocar, de forma aleatória, as crianças nos dois grupos, manter salas separadas para tratamento e avaliação e padronizar o momento de avaliação para 15 minutos após o início da inalação nos dois grupos - tempo necessário para finalizar a nebulização. A avaliação processou-se, portanto, 15 minutos após a dose no grupo IDE e imediatamente após a dose no NEB; no entanto, considerando que a maior parte da dose é liberada nos primeiros 5 minutos da nebulização - até cerca de $85 \%$ da dose, com alguns nebulizadores ${ }^{24}$ a avaliação ocorreu cerca de 10 minutos após a liberação da maior parte da dose no grupo NEB e 15 minutos após a liberação da dose no grupo IDE, diferença de tempo que não deve ter interferido nos resultados.

A tabela de avaliação foi criada para este trabalho, com base na tabela de Wood e Downes ${ }^{25}$.

A freqüência cardíaca maior no grupo NEB, no T0, pode ter sido decorrente de uma discreta predominância de lactentes nesse grupo. Como foi usada para comparação entre os grupos a pontuação global, e não os pontos conferidos a cada parâmetro clínico, a diferença no item coloração da pele no T0 (maior pontuação no grupo NEB) foi desconsiderada, por não ter comprometido a classificação da gravidade. Observou-se uma maior variabilidade nas pontuações do grupo IDE, no tempo $0 \mathrm{e}$, ao contrário, uma maior variabilidade de pontuação no NEB, após as inalações. Isso demonstra o efeito mais previsível e consistente do broncodilatador quando ministrado em aerossol dosimetrado com espaçador (menor dispersão de resultados). Não foram encontradas diferenças significativas nas pontuações pós-salbutamol, entre os dois grupos. No entanto, a menor necessidade de corticosteróide no grupo IDE $(\mathrm{p}=0,076)$ pode ser um indício de uma maior eficiência do dispositivo inalatório (aerossol dosimetrado com espaçador), levando a uma maior penetração do broncodilatador nas vias aéreas inferiores, ou de que tenha havido uma concentração, ao acaso, no grupo NEB, de pacientes com processo inflamatório brônquico maior, o que não parece provável em razão do método aleatório de alocação das crianças nos grupos; a demonstração da equivalência de doses clínicas diferentes pode também sugerir uma maior eficiência terapêutica do dispositivo IDE, com o qual foi utilizado apenas $1 / 3$ da dose usada com o nebulizador. O poder do estudo foi pequeno (Tabela 2), indicando que seria necessário estudarem-se amostras bem maiores para poder evidenciar diferenças significativas.

A utilização de doses diferentes do salbutamol nos dois grupos está de acordo com a rotina praticada no setor de pronto-atendimento do CPPHO, baseada em estudos que mostram uma maior proporção de partículas depositadas em vias aéreas inferiores com o inalador dosimetrado com espaçador do que com o nebulizador ${ }^{26,27}$. Wildhaber ${ }^{27}$ mostra que o inalador dosimetrado acoplado a dois tipos de espaçadores disponibiliza para inalação uma maior quantidade de partículas (40,2\% e 40,7\% das partículas geradas) que o nebulizador $(25,3 \%)$. Os estudos revistos (Tabela 6), que compararam nebulizador com aerossol dosimetrado acoplado a espaçador ${ }^{14,18-23}$, também utilizaram doses diferentes nos dois sistemas inalatórios (4 a 37 vezes maior com o nebulizador, conforme o estudo). Um trabalho em modelo de pulmão neonatal em ventilação mecânica ${ }^{23}$ mostrou a equivalência entre $100 \mu \mathrm{g}$ (através de aerossol dosimetrado com espaçador) e 2.500 a $3.700 \mu \mathrm{g}$ (através de nebulizador), medindo a quantidade de droga depositada nas vias aéreas inferiores centrais. Assim como no presente estudo, os demais trabalhos revistos (Tabela 6) não encontraram diferenças significativas na resposta ao tratamento ministrado pelos dois dispositivos inalatórios; alguns desses trabalhos utilizaram pontuação clínica e oximetria, como o presente estudo, e outros usaram testes de função pulmonar. A comparação entre a dose de $200 \mu \mathrm{g}$ versus 600 a $1.000 \mu \mathrm{g}$, sempre através de inalador dosimetrado e espaçador ${ }^{18}$, também não mostrou diferença significativa, indicando que doses pequenas são suficientes, e o efeito terapêutico não aumenta com a elevação da dose.

$\mathrm{O}$ presente estudo evidenciou uma diferença muito significativa entre os custos, vantajosa para o tratamento com inalador pressurizado e espaçador caseiro, sendo esse um resultado importante e que está em concordância com outros estudos 20,28 .

Não se observou preferência das crianças e suas famílias sobre o tipo de inalador utilizado, diferentemente do que relata outro estudo ${ }^{20}$, no qual os pais preferiram o inalador dosimetrado com espaçador. Provavelmente o baixo nível intelectual dos familiares dificultou essa investigação. $\mathrm{O}$ 
Tabela 6 - Trabalhos comparando diferentes dispositivos inalatórios, para uso de broncodilatador, por crianças

\begin{tabular}{|c|c|c|c|c|c|}
\hline Autores & Dispositivos & $\begin{array}{l}\text { Parâmetros } \\
\text { avaliados }\end{array}$ & $\begin{array}{l}\text { Doses do } \\
\text { broncodilatador }\end{array}$ & $\begin{array}{l}\mathrm{N}^{\circ} \text { de crianças } \\
\text { e gravidade }\end{array}$ & Resultados \\
\hline $\begin{array}{l}\operatorname{Zar}^{15} \\
1999\end{array}$ & $\begin{array}{l}\text { Espaçadores } \\
\text { convencionais } \mathrm{x} \\
\text { garrafa selada } \mathrm{x} \\
\text { garrafa não selada x copo } \\
\text { (unilateralmente cego, } \\
\text { randomizado }\end{array}$ & $\begin{array}{l}\text { Pontuação clínica, } \\
\text { oximetria, função } \\
\text { pulmonar (VEF1, } \\
\text { PEF) }\end{array}$ & $\begin{array}{l}400 \mu \mathrm{g}(<25 \mathrm{~kg}) \\
600 \mu \mathrm{g}(>25 \mathrm{~kg}) \\
\text { Só } 1 \text { dose }\end{array}$ & $\begin{array}{l}88 \text { crianças } \\
\text { Crise leve } \\
\text { e moderada } \\
\text { a grave }\end{array}$ & $\begin{array}{l}\text { Em crise leve: sem diferenças } \\
\text { entre os espaçadores. } \\
\text { Em crise moderada a grave: } \\
\text { efeitos com espaçador } \\
\text { convencional > garrafa selada > } \\
\text { garrafa não selada > copo }\end{array}$ \\
\hline $\begin{array}{l}\text { Teo }^{13} \\
1988\end{array}$ & $\begin{array}{l}\text { Garrafa plástica: } \\
\text { grupo salbutamol x } \\
\text { grupo placebo }\end{array}$ & PEF & $\begin{array}{l}500 \mu \mathrm{g} \\
\text { Só } 1 \text { dose }\end{array}$ & $\begin{array}{l}22 \text { crianças } \\
\text { Crise leve a } \\
\text { moderada }\end{array}$ & $\begin{array}{l}\text { Garrafa funciona bem } \\
\text { como espaçador }\end{array}$ \\
\hline $\begin{array}{l}\text { Wildhaber }^{14} \\
1999\end{array}$ & $\begin{array}{l}\text { ID e garrafa } \mathrm{x} \\
\text { nebulizador } \\
\text { (estudo cruzado, } \\
\text { randomizado) }\end{array}$ & $\begin{array}{l}\text { Deposição de } \\
\text { salbutamol } \\
\text { (gama câmara) }\end{array}$ & $\begin{array}{l}400 \mu \mathrm{g} \text { - inalador } \\
\text { dosimetrado } \\
2.000 \mu \mathrm{g}- \\
\text { nebulizador }\end{array}$ & $\begin{array}{l}17 \text { crianças } \\
\text { em intercrise }\end{array}$ & $\begin{array}{l}\text { Proporção de droga depositada } \\
\text { nos pulmões semelhante com } \\
\text { os dois dispositivos inalatórios }\end{array}$ \\
\hline $\begin{array}{l}\text { Schuh }^{18} \\
1999\end{array}$ & $\begin{array}{l}\text { ID + Aerochamber x } \\
\text { nebulizador } \\
\text { (duplo cego, } \\
\text { randomizado) }\end{array}$ & $\begin{array}{l}\text { Pontuação clínica } \\
\text { Oximetria } \\
\text { Frequêência respira- } \\
\text { tória } \\
\text { VEF1 }\end{array}$ & $\begin{array}{l}\text { ID: } \\
600 \text { a } 1.000 \mu \mathrm{g} \text { ou } \\
200 \mu \mathrm{g} \\
\text { Nebulizador: } \\
150 \mu \mathrm{g} / \mathrm{kg}\end{array}$ & $\begin{array}{l}90 \text { crianças } \\
\text { em crise } \\
\text { asmática leve }\end{array}$ & $\begin{array}{l}\text { Não houve diferenças entre } \\
\text { os três grupos }\end{array}$ \\
\hline $\begin{array}{l}\text { Garner }^{19} \\
2002\end{array}$ & $\begin{array}{l}\text { ID + espaçador x } \\
\text { nebulizador } \\
\text { (cruzado, cego, } \\
\text { randomizado) }\end{array}$ & $\begin{array}{l}\text { Mecânica respirató- } \\
\text { ria passiva e } \\
\text { Concentração sérica } \\
\text { de salbutamol }\end{array}$ & $\begin{array}{l}400 \mu \mathrm{g}(\mathrm{ID}) \mathrm{e} \\
150 \mu \mathrm{g} / \mathrm{kg}(\mathrm{Neb}) \\
\text { separadas por } 4 \text { horas }\end{array}$ & $\begin{array}{l}12 \text { lactentes } \\
\text { intubados, } \\
\text { em ventilação } \\
\text { mecânica }\end{array}$ & $\begin{array}{l}\text { Não houve diferenças } \\
\text { significativas }\end{array}$ \\
\hline $\begin{array}{l}\text { Leversha }^{20} \\
2000\end{array}$ & $\begin{array}{l}\text { ID +Aerochamber } \\
\mathrm{x} \\
\text { nebulizador } \\
\text { (duplo cego, } \\
\text { randomizado, } \\
\text { controlado } \\
\text { com placebo) }\end{array}$ & $\begin{array}{l}\text { Pontuação clínica } \\
\text { Oximetria } \\
\text { Freq. respiratória } \\
\text { Freqüência cardíaca } \\
\text { Ausculta respiratória } \\
\text { Custos dos } \\
\text { tratamentos }\end{array}$ & $\begin{array}{l}600 \mu \mathrm{g} \text { de salbutamol } \\
\text { (ID) + placebo (neb) } \\
\mathrm{e} \\
2.500 \mu \mathrm{g} \text { de } \\
\text { salbutamol (neb) } \\
\text { + placebo (ID) } \\
\text { Doses repetidas de 20/ } \\
20 \text { min (máximo de } 6 \text { ) }\end{array}$ & $\begin{array}{l}60 \text { crianças de } \\
1 \text { a } 4 \text { anos, em } \\
\text { crise asmática } \\
\text { moderada a } \\
\text { grave }\end{array}$ & $\begin{array}{l}\text { ID causou maior redução da } \\
\text { sibilância, menor elevação da } \\
\text { frequência respiratória e teve } \\
\text { menor custo. } \\
\text { Demais parâmetros, sem } \\
\text { diferença significativa }\end{array}$ \\
\hline $\begin{array}{l}\text { Rubilar } \\
2000\end{array}$ & $\begin{array}{l}\text { ID + espaçador + } \\
\text { máscara } \mathrm{x} \\
\text { nebulizador } \\
\text { (unilateralmente } \\
\text { cego, randomizado }\end{array}$ & Pontuação clínica & $\begin{array}{l}200 \mu \mathrm{g} \text { de salbutamol } \\
\text { de } 10 / 10 \text { min, } 5 \text { vezes } \\
\text { (ID) } \\
250 \mu \mathrm{g} / \mathrm{kg} \text { de } \\
\text { salbutamol } \\
\text { de } 13 / 13 \mathrm{~min}, \\
3 \text { vezes (neb) }\end{array}$ & $\begin{array}{l}123 \text { lactentes de } \\
1 \text { a } 24 \text { meses) } \\
\text { com crise de } \\
\text { sibilância } \\
\text { moderada } \\
\text { a grave }\end{array}$ & $\begin{array}{l}\text { Resposta mais rápida com o ID: } \\
\text { na primeira hora de tratamento, } \\
\text { houve boa resposta em } 90 \% \text { do } \\
\text { grupo ID e em } 71 \% \text { do grupo neb. } \\
\text { Após } 2 \text { horas, em } 100 \% \text { do grupo } \\
\text { ID e em } 94 \% \text { do grupo neb. }\end{array}$ \\
\hline $\begin{array}{l}\text { Closa }^{22} \\
1998\end{array}$ & $\begin{array}{l}\text { ID + espaçador } \mathrm{x} \\
\text { nebulizador } \\
\text { (duplo cego, } \\
\text { randomizado) }\end{array}$ & $\begin{array}{l}\text { Pontuação clínica } \\
\text { Oximetria }\end{array}$ & $\begin{array}{l}500 \mu \mathrm{g} \text { de terbutalina, } \\
2 \text { vezes ID) } \\
2.000 \mu \mathrm{g} \text { de terbutali- } \\
\text { na, } 2 \text { vezes (neb) }\end{array}$ & $\begin{array}{l}34 \text { lactentes de } \\
1 \text { a } 24 \text { meses } \\
\text { em crise de } \\
\text { sibilância }\end{array}$ & Sem diferença significativa. \\
\hline $\begin{array}{l}\text { Lugo }^{23} \\
2001\end{array}$ & $\begin{array}{l}\text { ID }(\text { com CFC })+ \\
\text { espaçador } \\
\text { ID }(\text { com HFA })+ \\
\text { espaçador } \\
\text { Nebulizador }\end{array}$ & $\begin{array}{l}\text { Dosagem do salbu- } \\
\text { tamol coletado no } \\
\text { fim do tubo endotra- } \\
\text { queal }\end{array}$ & Salbutamol & $\begin{array}{l}\text { Modelo } \\
\text { neonatal de } \\
\text { ventilação } \\
\text { pulmonar }\end{array}$ & $\begin{array}{l}\text { Salbutamol dosado com ID foi } \\
\text { significativamente maior que } \\
\text { salbutamol dosado com nebuliza- } \\
\text { dor. } 100 \mu \mathrm{g} \text { por ID equivaleram a } \\
2.500 \text { a } 3.700 \mu \mathrm{g} \text { por nebulizador }\end{array}$ \\
\hline $\begin{array}{l}\text { Singhal } \\
2001\end{array}$ & $\begin{array}{l}\text { ID + espaçador } \\
\text { convencional } \\
\text { ID + espaçador artesanal } \\
\text { (unilateralmente cego, } \\
\text { randomizado) }\end{array}$ & $\begin{array}{l}\text { PEF } \\
\text { Oximetria } \\
\text { Freq. respiratória } \\
\text { Frequiência cardíaca }\end{array}$ & Salbutamol & 60 & Sem diferença significativa \\
\hline
\end{tabular}


tempo para veiculação do broncodilatador no IDE, significativamente menor que no NEB, deve influir na tolerância do método pelas crianças e na preferência da família, embora este estudo não tenha sido capaz de revelar isso.

Algumas pequenas evidências de vantagem do aerossol dosimetrado com espaçador foram apontadas por outros autores, porém não investigadas neste estudo, como: resposta mais rápida ${ }^{21}$, menor número de admissões ${ }^{20}$, melhora mais intensa da sibilância e menor aumento da frequência cardíaca $^{20}$, com diferenças significativas.

A terapia inalatória da asma em crianças é um tema de grande importância; é preciso definir doses e métodos mais adequados. O presente trabalho traz contribuição significativa, ao analisar o desempenho de um espaçador simples e de custo zero. Os resultados deste estudo estão de acordo com o que vem sendo reportado na literatura. Considerando as menores doses usadas com o inalador dosimetrado e espaçador, este trabalho demonstra a maior eficiência clínica deste dispositivo em relação ao nebulizador - método tradicional e ainda amplamente utilizado em pediatria. Esse estudo mostra uma comparação de custos entre as duas modalidades terapêuticas, que favorece também o inalador dosimetrado. Os resultados respaldam a recomendação da utilização preferencial do aerossol dosimetrado com espaçador artesanal em relação ao nebulizador, na administração de broncodilatador a crianças de qualquer idade, em crise de asma moderada, e justificam que se proponha revisão nas rotinas de tratamento da crise asmática nas enfermarias e serviços de emergência pediátricos.

\section{Referências bibliográficas}

1. Guidelines for the diagnosis and management of asthma. National Asthma Education and Prevention Program - National Heart, Lung and Blood Institute Information Center [s.1]. Meeting of the American Academy of Asthma and Immunology; 1997. p. 50.

2. II Consenso Brasileiro no Manejo da Asma. Sociedade Brasileira de Alergia e Imunopatologia, Sociedade Brasileira de Pediatria e Sociedade Brasileira de Pneumologia e Tisiologia. J Pneumologia 1998;24:171-276.

3. Taburet AM, Schmit B. Pharmacokinetic optimisation of asthma treatment. Clin Pharmacokinet 1994;26:396-418.

4. O'Callaghan C, Milner AD, Swarbrick A. Paradoxical deterioration in lung function after nebulised salbutamol in wheezy infants. Lancet 1986; 2(8521-22):1424-5.

5. Souza LSF. Aerossolterapia na asma da criança. J Pediatr (Rio J) 1998;74:189-204.

6. Tal A. Deposition pattern of radiolabeled salbutamol inhaled from a metered-dose inhaler by means of a spacer with mask in young children with airway obstruction. J Pediatr 1996;128:479-84.
7. Newman SP, Clarke SW. Improvement of drug delivery with a breath actuated pressurized aerosol for patients with poor inhaler technique. Thorax 1991;46:712-16.

8. Keeley D. Large volume plastic spacers in asthma should be used more. BMJ 1992;305:598-9.

9. Barry PW, O'Callaghan CO. In vitro comparison of the amount of salbutamol available for inhalation from different formulations used with different spacer devices. Eur Respir J 1997; 10:1345-8.

10. Chege JK, Chrystyn H. Volumatic usage: some generic salbutamol metered-dose inhalers can be used. Thorax 1994;49:1162-3.

11. Jackson C. Optimizing inhaled drug delivery in patients with asthma. Br J Gen Pract 1995;45:683-7.

12. Barry PW, Robertson CF, O'Callaghan CO. Optimum use of spacer device. Arch Dis Child 1993;69:693-4.

13. Teo J, Kwang LW, Yip William CL. An inexpensive spacer for use with metered-dose bronchodilators in young asthmatic children. Pediatr Pulmonol 1988;5:244-6.

14. Wildhaber JH, Dore ND, Wilson JM, Devadason SG, LeSouef PN. Inhalation therapy in asthma: nebulizer or pressurized metered-dose inhaler with holding chamber? In vivo comparison of lung deposition in children. J Pediatr 1999;135:28-33.

15. Zar HJ, Brown G, Donson H, Brathwaite N, Mann MD, Weinberg EG. Home-made spacers for bronchodilator therapy in children with acute asthma: a randomized trial. Lancet 1999;354:979-82.

16. Singhal T, Garg H, Arora HS, Lodha R, Pandey RM, Kabra SK. Efficacy of a home-made spacer with acute exacerbation of bronchial asthma: a randomized controlled trial. Indian J Pediatr 2001;68:37-40.

17. Siegel S. Estatística não-paramétrica para as ciências do comportamento. São Paulo: McGraw-Hill; 1975. p. 350.

18. Schuh S, Johnson SW, Stephens D, Callahan S, Winders P, Canny GJ. Comparison of albuterol delivered by a metered-dose inhaler with spacer versus a nebulizer in children with mild acute asthma. J Pediatr 1999;135:22-7.

19. Garner SS, Wiest DB, Bradley JW, Habib DM. Two administration methods for inhaled salbutamol in intubated patients. Arch Dis Child 2002;87:49-53.

20. Leversha AM, Campanella SG, Aickin RP, Asher MI. Costs and effectiveness of spacer versus nebulizer in young children with moderate and severe acute asthma. J Pediatr 2000;136:428-31.

21. Rubilar L, Castro-Rodriguez JA, Girardi G. Randomized trial of salbutamol via metered-dose inhaler with spacer versus nebulizer for acute wheezing in children less than 2 years of age. Pediatr Pulmonol 2000;29:264-9.

22. Closa RM, Ceballos JM, Gomez-Papi A, Galiana AS, Gutierrez C, Marti-Henneber C. Efficacy of bronchodilators administered by nebulizers versus spacer devices in infants with acute wheezing. Pediatr Pulmonol 1998;26:344-8.

23. Lugo RA, Kenney JK, Keenan J, Salyer JW, Ballard J, Ward RM. Albuterol delivery in a neonatal ventilated lung model: nebulization versus chlorofluorocarbon- and hydrofluoroalkanepressurized metered dose inhalers. Pediatr Pulmonol 2001;31:247-54.

24. O'Callaghan C. How to get drugs into the respiratory tract. Arch Dis Child 1993;68:441-3.

25. Wood D, Downes JJ, Lecks HT. A clinical scoring system for the diagnosis of respiratory failure. Preliminary report on childhood status asthmaticus. Am J Dis Child 1972;123:227-8.

26. Warner JO, Naspitz CK. Third International Pediatric Consensus Statement on the Management of Childhood Asthma. Pediatr Pulmonol 1998;25:1-17. 
27. Wildhaber JH, Devadason SG, Hayden MJ, Eber E, Summers QA, Le-Souef PN. Aerosol delivery to wheezy infants: a comparison between a nebulizer and two small volume spacers. Pediatr Pulmonol 1997;23:212-16.

28. Orens DK, Kester L, Fergus LC, Stoller JK. Cost impact of metered-dose inhalers vs. small volume nebulizers in hospitalized patients: The Cleveland Clinic experience. Respir Care 1991;36:1099-114.
Endereço para correspondência:

Dra. Liana Vilarinho

Rua João Bião de Cerqueira, 212 - ap. 502 - Pituba CEP 41830-580 - Salvador - BA

Tel.: (71) 351.8057

E-mail:lianavilarinho@uol.com.br 